\title{
Warburg Effect and Cancer Survival
}

\author{
William Panosyan ${ }^{1}$, Daniel Panosyan ${ }^{1}$ and Eduard Panosyan\# \\ ${ }^{1}$ Cresenta Valley High School, La Cresenta, CA, USA \\ \#Advisor
}

ABSTRACT

Warburg effect is a major metabolic shift of glycolysis in cancer cells towards anaerobic fermentation. Key reaction here overproduces lactate from pyruvate catalyzed by lactate dehydrogenase-A (LDHA). Augmented Warburg effect and oncometabolism may contribute to cancer progression worsening patients' survival. Expressions of various key enzymes were used as surrogates to validate the clinical influence of metabolic alterations in cancers. Expression and Kaplan-Meier survival data were extracted from the R2 Genomics Analysis and Visualization Platform (R2) and Human Protein Atlas (HPA). Expression of LDHA gene was 2-4 times higher than normal tissues in certain cancers including breast, colon, genitourinary and B-cell lymphomas. No cancer specificity or strong associations were observed between RNA and protein expressions of LDHA. However, higher LDHA gene expression correlated with poorer survival in renal, liver, lung, pancreatic, cervical and breast cancers. These cancers moderately or strongly stained for LDHA protein. Per HPA, gliomas had low LDHA without survival correlation. Nevertheless, this correlation was observed in the largest glioblastoma database (R2). Furthermore, 3 of 4 medulloblastoma subtypes showed poor survival with higher LDHA. Contrarily, in B-cell lymphomas and colon cancer high LDHA was a favorable prognostic marker. Poor survival correlated with high expression of other enzymes for glycolysis and amino acid metabolism (PFK-isoenzymes, GFPT2 and BCAT1). Warburg effect may not be universally dominant for all cancers, but most cancers have high LDHA and/or associated poor survival - confirming importance of this metabolic derangement in cancers. Heterogeneity of metabolic alterations can serve to diversify anti-metabolic strategies for targeted anti-cancer therapies.

\section{Introduction}

Warburg effect refers to a well-known metabolic reprogramming that excessively shifts glycolysis in cancer cells towards anaerobic fermentation (Koukourakis \& Giatromanolaki, 2019; Potter, Newport, \& Morten, 2016; Warburg, Wind, \& Negelein, 1927). Key reaction here overproduces lactate from the pyruvate catalyzed by an enzyme called lactate dehydrogenase (LDH) (Koukourakis \& Giatromanolaki, 2019). It contributes in cell proliferation and cancer progression (Feng et al., 2018; Valvona, Fillmore, Nunn, \& Pilkington, 2016), Figure 1. One of our aims in this research was to learn about the potential influence of the Warburg effect on cancer survival, which would support to validate the clinical relevance for this phenomenon of oncometabolism in cancers. Other overexpressed key metabolic enzymes may have similar clinical relevance.

We hypothesized that the gene and/or protein expressions of key molecule(s) in cellular metabolism such as the main enzyme(s) LDHA will be higher in cancer tissues and/or will correlate with poor survival of patients with variable cancer types. Key enzymes other than LDHA, which reportedly are overexpressed in several different types of cancers, also were considered in our research. These included PFK (Phosphofructokinase), which sustains high rate of glycolysis (Hasawi, Alkandari, \& Luqmani, 2014), GFPT2 (Glutamine-fructose-6-phosphate transaminase 2) which is one of the key enzymes for hexosamine biosynthesis (Akella, Ciraku, \& Reginato, 2019), and BCAT1 (Branchedchain amino acid transaminase 1), converting BCAAs to BCKAs (Ananieva \& Wilkinson, 2018), Figure 1. 


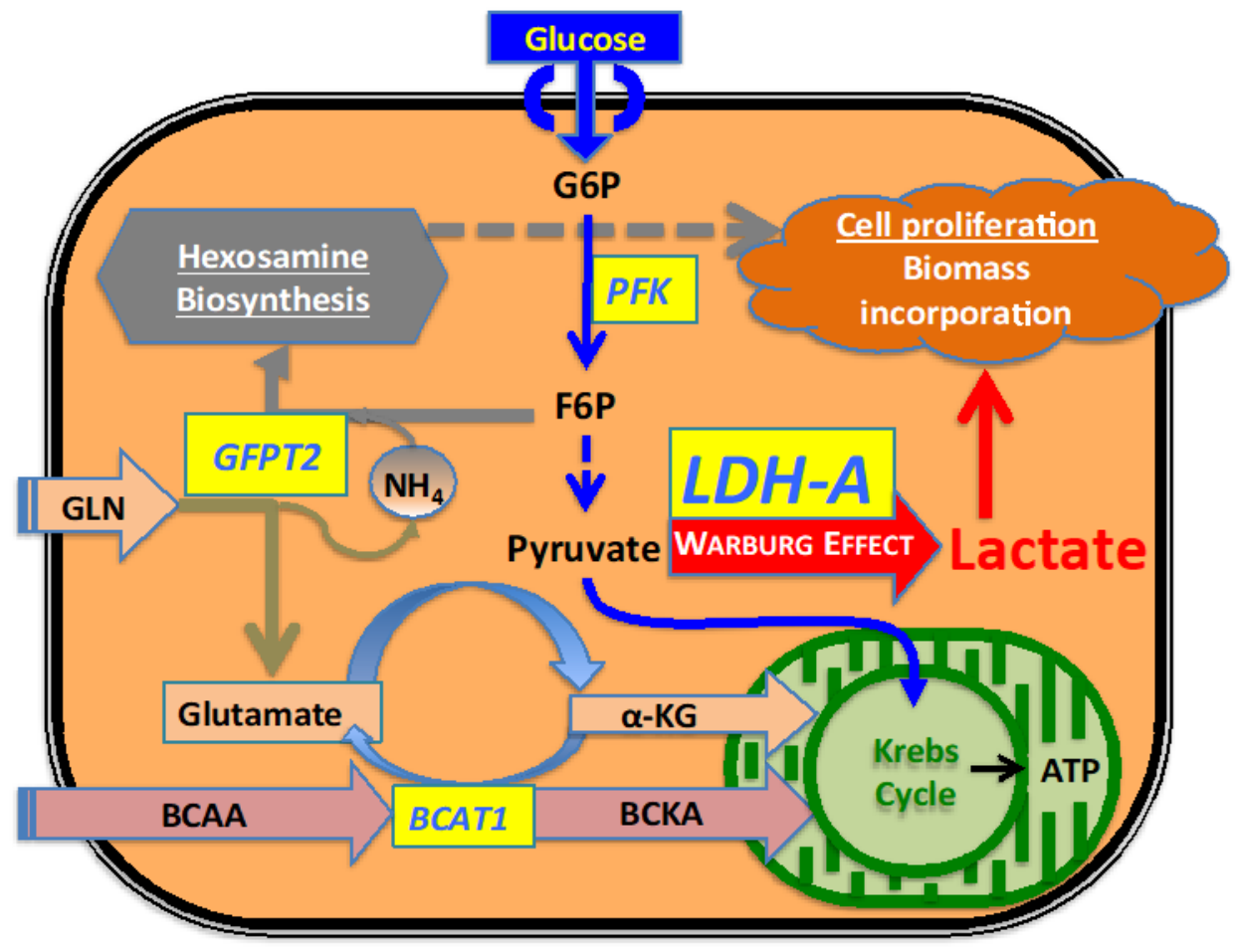

Figure 1. Oncometabolic interplay between the glycolysis, Warburg effect, hexosamine biosynthesis, branched chain amino acid metabolism, and Krebs cycle.

Metabolic reprogramming with Warburg effect shifts glycolysis in cancer cells towards excessive lactate formation from pyruvate (Koukourakis \& Giatromanolaki, 2019; Potter et al., 2016; Warburg et al., 1927). Key reaction here is catalyzed by lactate dehydrogenase A (LDHA). It contributes in cell proliferation and cancer progression (Feng et al., 2018; Koukourakis \& Giatromanolaki, 2019; Valvona et al., 2016). Other key overexpressed enzymes in different types of cancers (also depicted in yellow boxes) include:

- PFK (Phosphofructokinase), which sustains high rate of glycolysis (Hasawi et al., 2014),

- GFPT2 (Glutamine-fructose-6-phosphate transaminase 2) which is one of the key enzymes for hexosamine biosynthesis (Akella et al., 2019), and

- BCAT1 (Branched-chain amino acid transaminase 1), converting BCAAs to BCKAs (Ananieva \& Wilkinson, 2018).

Other abbreviations:

$\alpha$-KG: Alpha-ketoglutarate

ATP: Adenosine triphosphate

BCAA: Branched-chain amino acids

BCKA: Branched-chain keto acids

GLN: Glutamine 
G6P: Glucose 6-phosphate

F6P: $\quad$ Fructose 6-phosphate

\section{Materials and Methods}

Enzymatic expressions at gene and protein levels (primarily for LDHA) were used as indirect surrogates to measure the potential influence of the metabolic alterations related to Warburg effect in various cancers. Expression data and Kaplan-Meier survival plots were extracted from websites for R2 Genomics Analysis and Visualization Platform (R2)((http://r2.amc.nl)) and Human Protein Atlas (HPA, Atlas) including data from The Cancer Genome Atlas (TCGA). Descriptive statistics was used for data analysis.

Gene expressions in normal versus cancer tissues were extracted for comparison by using a corresponding feature in R2 platform (mega-sampler technique across many datasets for a single gene - in this case LDHA). Datasets containing more than 200 samples were selected for comparing LDHA expression in various normal versus cancer tissues. For B cell -lymphomas, all the datasets with more than 20 samples were included. Box plots with standards errors were used to generate the graphs, which were color-coded for better distinction of normal versus tumor datasets. Gene versus protein expression data for LDHA in 17 types of cancer was obtained from HPA site (Atlas). The RNA Expression levels for LDHA gene were aligned against the percentages of positively stained samples of LDHA protein per cancer type (as correspondingly shown in upper and lower panels in Figure 3). For display of LDHA protein immunohistochemical (IHC) staining, version with CAB069404 was selected.

Automated analytic features in HPA and R2 sites were used to generate Kaplan-Meier (KM) survival plots for a given single gene at a time. For HPA, single gene entry provides selected types of cancers with prognostic significance of the given molecule with $\mathrm{p}$ value of $\mathrm{P}<0.001$ (Atlas) (as shown in upper 5 panels/cancer types of Figure 4 in relation to LDHA gene, and Table 1 for all the genes/cancers except GBM). For Kaplan-Meier plots from R2 (http://r2.amc.nl), single dataset analysis was conducted individually for each gene, all using P-values $<0.05$ as a cutoff for significant survival differences (such as for the breast cancer and GBM in last two panels of Figure 4). Additional KM analyses were conducted for other selected enzymes of interest that included PFK isoenzymes, GFPT2 and BCAT1. 


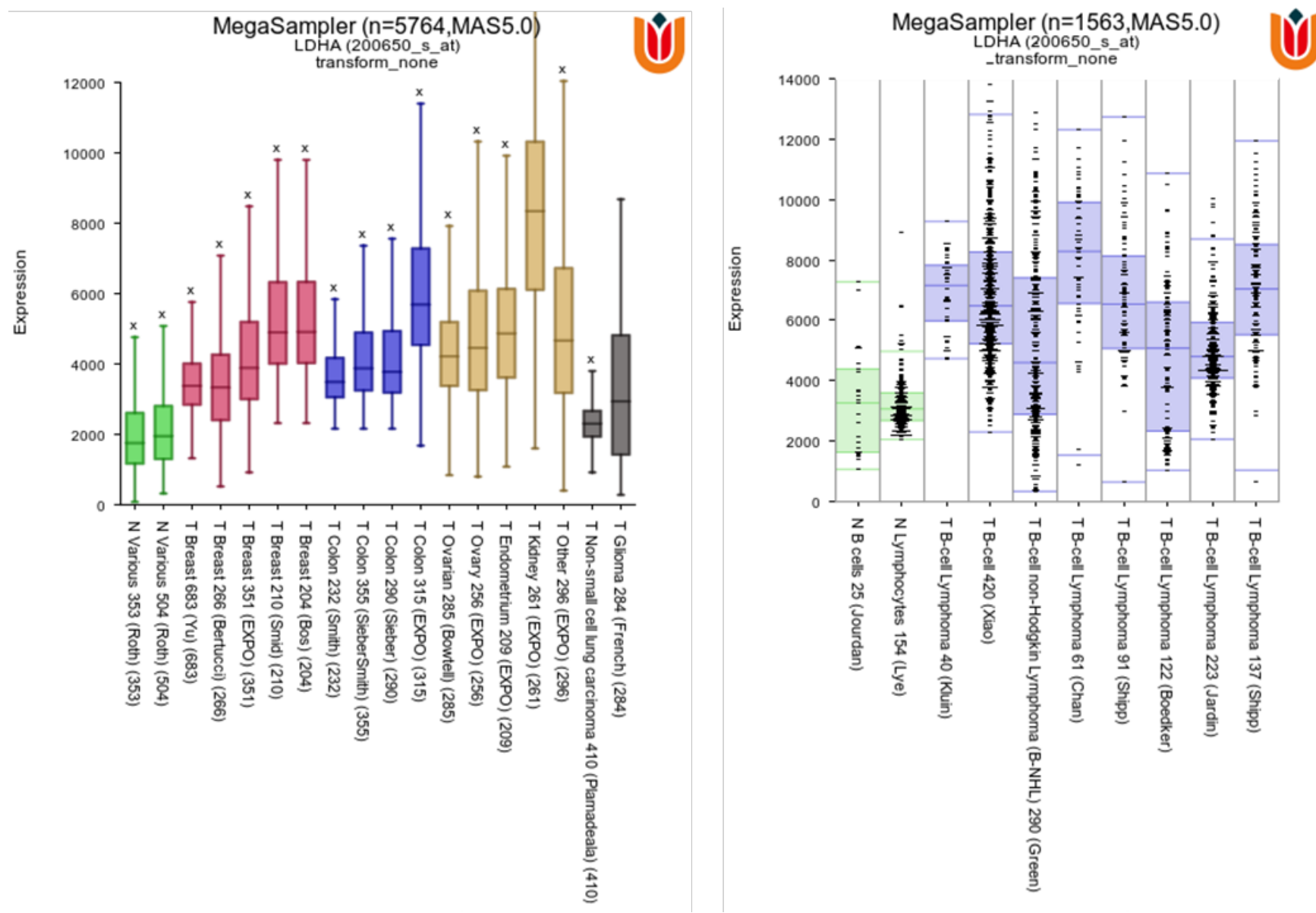

Figure 2. Expression of LDHA gene across several normal and cancer tissue datasets from R2: Genomics Analysis and Visualization Platform (http://r2.amc.nl).

Left sided graph demonstrates box plots with standards errors for LDHA expression in selected datasets with more than 200 samples: 2 normal tissue datasets (various tissues, in green) and 16 tumor datasets. It shows in average 2-4 times higher expression of LDHA in majority of cancer datasets, with exception of ones for NSCLC and glioma (last 2 gray sets). Data from breast cancer datasets are color-coded with pink, colon cancer - with blue, and grouped genitourinary and other cancers - with yellow.

Right-sided graph shows dot-box plots of LDHA expression in normal B-cells and Lymphocytes (first two sets in light green), which in general are 2-fold lower than the expression in 8 datasets of B-cell lymphoma (depicted in light purple).

\section{Results}

Initial results showed wide variability in expression of LDH-A gene across the multiple cancer datasets in R2. Comparison of LDHA gene expression in normal versus cancer tissues demonstrated 2-4 fold higher levels in certain cancer groups such as breast (5 datasets), colon (4 datasets), genitourinary (4 datasets), and in a dataset with other cancer types (Figure 2). Nevertheless, as exemplified in the last two bars of the left-sided panel in Figure 2, not all cancer types showed increased LDHA gene expression: NSCLC (non-small cell lung cancer) and gliomas had expressions comparable to normal tissues. Further, two datasets of normal B-cells and lymphocytes showed LDHA gene slightly 
elevated above the various normal tissues, however it largely appeared twice lower than in most of the 8 B-lymphoma datasets, as shown in the right-sided graph of Figure 2.

Alongside with low cancer specificity, association between the RNA and protein expression levels for LDHA was not very strong as seen in lower and upper panels of Figure 3. For example, Pancreatic and Renal cancers had identical protein staining percentages, when the pattern of gene expression was different between two. On the other hand, while LDHA gene expression in Head and Neck cancers appeared similar to (if not higher than) Stomach cancer, protein expression levels actually showed the opposite pattern (Figure 3). However, higher LDH-A gene expression significantly correlated with poorer outcomes in many of cancer types that showed moderate to strong positivity for cytoplasmic, membranous and/or nuclear staining of the LDH-A protein (these included renal, liver, lung, pancreatic, cervical and breast cancers, Figure 4). In certain types of grouped cancers with relatively low LDH-A expression such as gliomas (Figures 2 and 3), there was no survival correlation, which nevertheless was observed in a population of patients with relevant brain tumors such as glioblastoma (Figure 4, last graph).

Furthermore, in 3 out of 4 subtypes of pediatric medulloblastoma tumors showed poor survival link with higher LDH-A expression (Figure 5). Interestingly, in certain cancers LDHA played out as a favorable prognostic marker including lymphoma (Guinney-3232 dataset in R2) and colon cancer (Xiao-420 dataset in R2).((http://r2.amc.nl))

Additionally, we exemplified poor survival associations with high expression of other genes coding for enzymes related to glycolysis and amino acid metabolism such as PFK isoenzymes, GFPT2 and BCAT1 (Table 1).
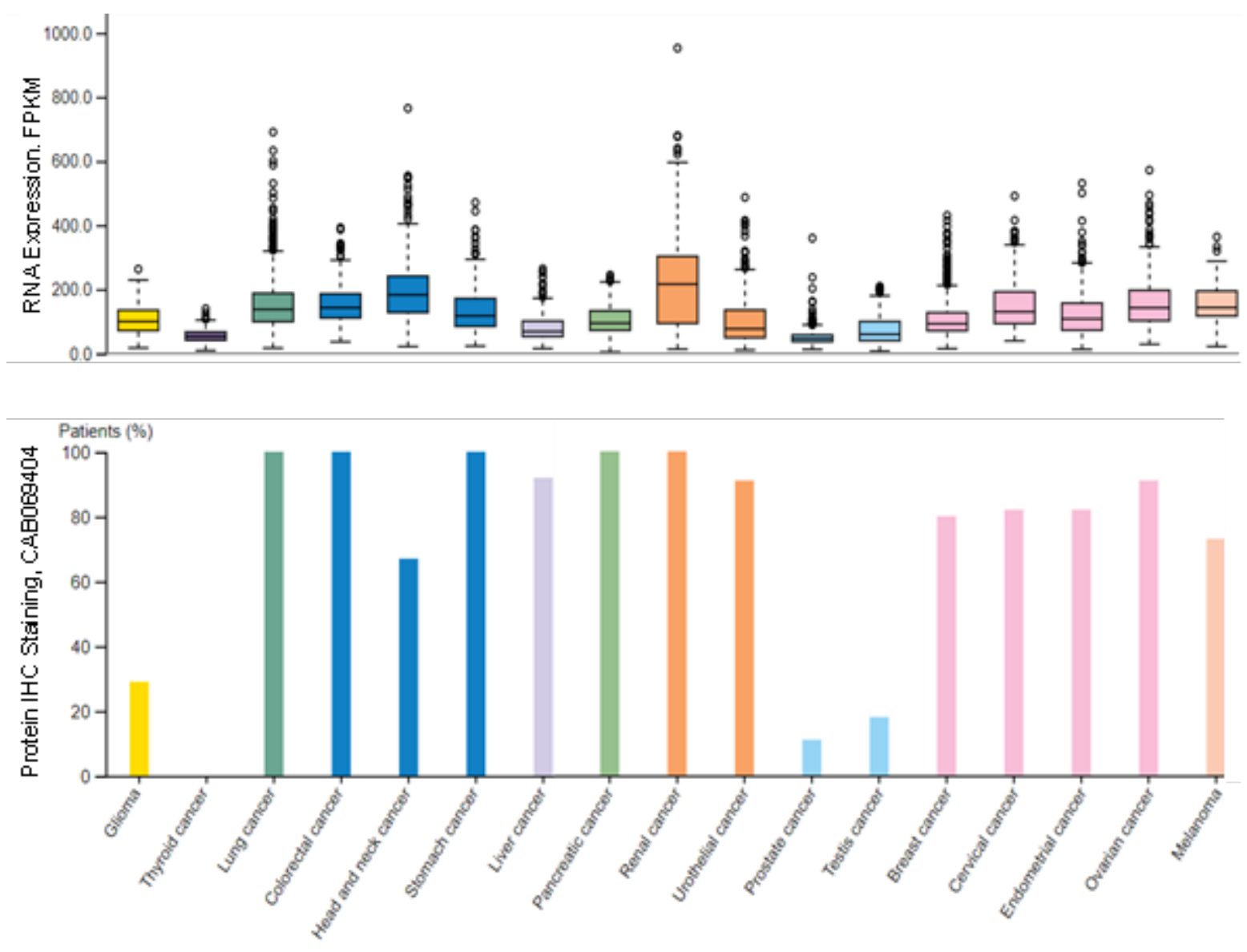

Figure 3. LDHA Gene (upper panel) and Protein (lower panel) expressions in 17 types of cancer tissues per data from Human Protein Atlas website (Atlas). 
Upper panel shows box plots for RNA expression across the 17 cancer types reported as median FPKM (number Fragments Per Kilobase of exon per Million reads) and generated by The Cancer Genome Atlas (TCGA). Data was based on mRNA expression levels across all grouped cancer tissues. Normal distribution across the dataset is visualized with box plots, shown as median and $25^{\text {th }}$ and $75^{\text {th }}$ percentiles. Points are displayed as outliers if they are above or below 1.5 times the interquartile range. The cancer types are color-coded according to cancer tissue origination.

Lower panel demonstrates percentages of patients whose samples showed a positive staining for LDHA protein in corresponding 17 types of cancer tissues. These displayed moderate to strong cytoplasmic positivity with additional membranous and/or nuclear staining. About $2 / 3$ of the cancer types showed more than $80 \%$ rate of positive immunohistochemical (IHC) staining (CAB069404). Most gliomas, thyroid, prostate and testicular cancers were weakly stained or negative. 


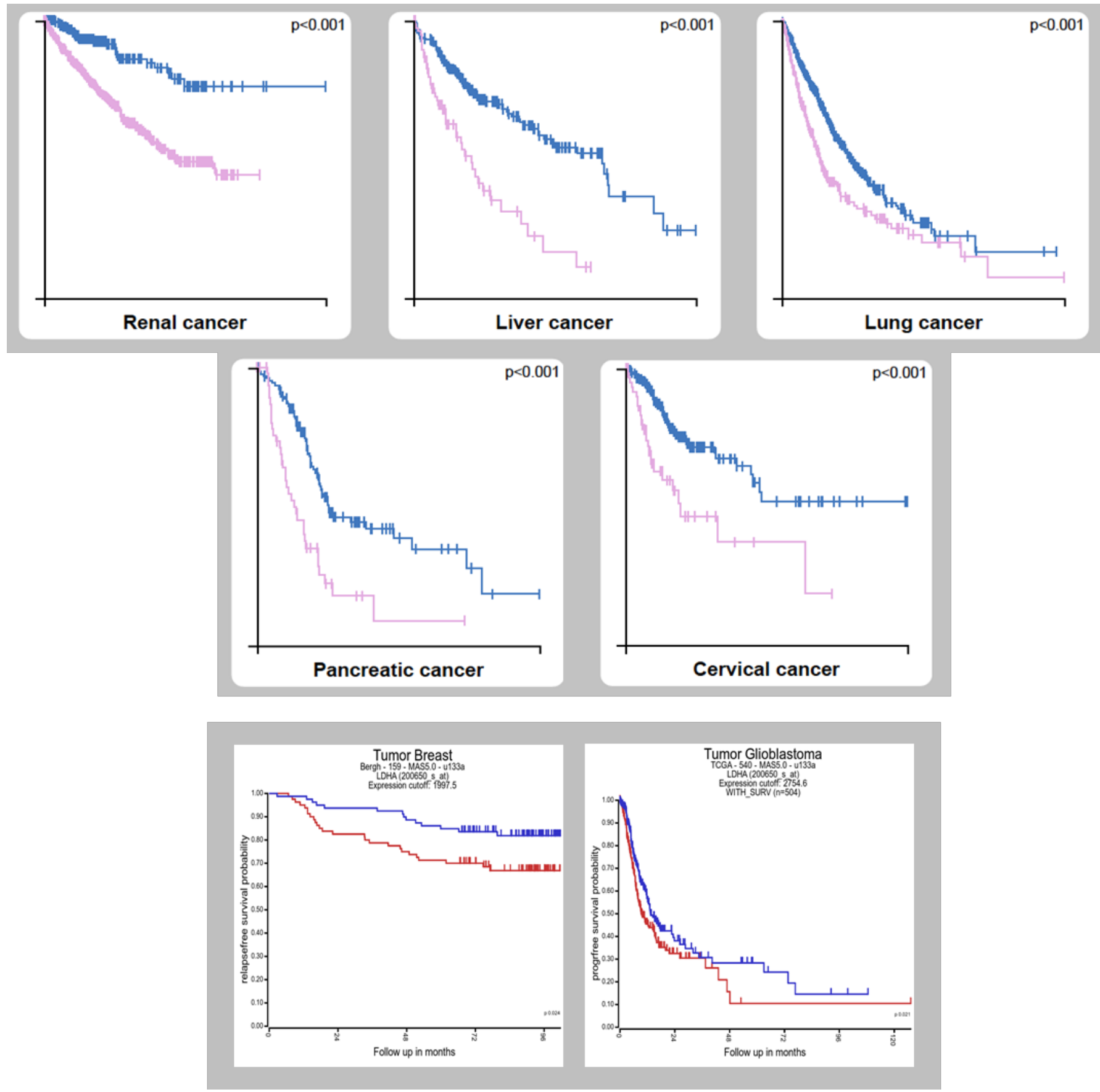

Figure 4. Kaplan-Meier plots for seven types of cancers where high expression of LDHA gene has significant association with patients' poor survival ((http://r2.amc.nl); Atlas).

The prognosis is unfavorable in all seven types of cancers with higher expression of LDHA (blue lines show high expression and pink/red lines show low expression). Upper 5 graphs were produced from Human Protein Atlas platform $(\mathrm{p}<0.001)$.

The $\mathrm{Y}$-axis shows the probability of survival (starts at $100 \%$, ends at $0 \%$ ), whereas $\mathrm{X}$-axis is time. 
Last 2 graphs were generated from R2 platform for breast cancer and GBM $(\mathrm{p}=0.02)$.

Table 1. Summary of associations between the poor survival and high gene expression for selected key enzymes in 12 types of cancer.

\begin{tabular}{|l|l|l|l|l|}
\hline \multirow{2}{*}{ Cancer type } & \multicolumn{4}{|l|}{ Enzymes the gene expression of which are associated with poor survival } \\
\cline { 2 - 5 } & LDHA & GFPT2 & BCAT1 & PFK isoenzymes* \\
\hline Cervical & + & + & & + (PFKFB4) \\
\hline Liver & + & & & + (PFKP) \\
\hline Lung & + & + & & \\
\hline Pancreatic & + & & & \\
\hline Renal & + & + & + & + (PFKFB3 \& PFKFB4) \\
\hline Head and Neck & & & + & + (PFKM \& PFKP) \\
\hline Stomach & & & + & \\
\hline Thyroid & & + & & + (PFKFB2) \\
\hline Urothelial & & & + & \\
\hline Endometrial & & & & $+($ PFKM) \\
\hline Ovarian & & & & $+($ PFKFB4) \\
\hline Glioblastoma & + & + & + & \\
\hline
\end{tabular}

Plus (+) signs depict poor survival with higher expression of the genes.

* PFK isoenzymes that are associated with survival are shown in parenthesis.

Survival associations were examined in a dozen of cancer types as shown in this table. In addition to LDHA, 3 other genes were examined that code for enzymes related to glycolysis (PFK isoenzymes), hexosamine biosynthesis (GFPT2) and amino acid metabolism (BCAT1). All the associations were extracted based on HPA site analyses, except GBM, which was from published R2/TCGA data (Panosyan et al., 2017).

This part of our work suggests that the heterogeneity of metabolic derangements can serve to diversify anti-metabolic strategies for developing more targeted therapies against various types of cancers.

\section{Discussion}

Cancer is a leading cause of death worldwide (Mukherjee, 16 November 2010). Uncontrolled cell proliferation of abnormal cancer cells in large is due to variety of genetic abnormalities (Mukherjee, 16 November 2010). Invasive growth of cancer tissues eventually results in host demise if therapies are not effective (Mukherjee, 16 November 2010). In addition to surgical removal of cancer tissues when possible, mainstream therapies are directed against cancer cell proliferation by the means of DNA damage (radiation and chemotherapy), cell-signaling inhibition, and (recently) by using immune-therapeutics (Mukherjee, 16 November 2010). An alternative, classic anti-metabolic strategy of killing hypermetabolic cancer cells by "starvation of a nutrient" was conceptualized after it was found that is hard to grow lymphoma cells on serum from guinea pigs. Dr. Broome published in 1963, that the enzyme cleaving the amino acid Asparagine (called Asparaginase) found in guinea pig serum was responsible for anti-lymphoma effect (Broome, 1963). Because certain lymphoma and leukemia cells are more sensitive to Asparagine depletion than normal cells, Asparaginase was later developed into a drug formulation (Ertel, Nesbit, Hammond, Weiner, \& Sather, 1979), and currently is an indispensible agent in armamentarium against many types of leukemia and certain lymphomas (Avramis \& Panosyan, 2005). This and other anti-metabolic strategies are appealing since they luck the side effects related to DNA damage, including potential ability to cause secondary cancers. 
Earlier, in 1920s doctors Warburg and Cori discovered another crucial metabolic aberration in cancer showing increased glucose usage and lactate formation by cancer cells (Koukourakis \& Giatromanolaki, 2019; Potter et al., 2016; Warburg et al., 1927). This well-described oncometabolic switch known as Warburg effect served the basis of developing contemporary diagnostic imaging modalities. Positron Emission Tomography (PET) measures radio-labeled glucose uptake increased in cancers (El-Galaly et al., 2018), and lactate is one of the main molecules evaluated on magnetic resonance spectroscopy (MRS) (Wilson et al., 2013). However, to date there are no clinically approved agents directly inhibiting Warburg effect. This in part is possibly due to the presence of anaerobic glycolysis in normal tissues, inhibition of which can possibly cause clinically significant side effects. Current pharmacological achievements theoretically provide an opportunity of targeted delivery of cytotoxic molecules to cancers while sparing normal tissues from the hit ("Antibody-drug Conjugate, a Potential Trojan Horse to Fight Cancer https://www.genscript.com/antibody-news/antibody-drug-conjugate-a-potential-trojan-horse-to-fight-cancer.html," May 18, 2021).

In this study, we primarily used the overexpression of LDHA and it's association with poor clinical outcome in variety of cancers to indirectly verify the clinical relevance of Warburg effect. LDHA expression showed low cancer specificity, and associations between the expressions of LDHA gene and protein for many cancer types are variable. Wide range of error bars in Figures 2 and 3 points to variability of LDHA expression within each cancer type, suggesting that Warburg effect may not be uniformly dominant in all cancers. Differences between the datasets of the same cancer type in Figure 2 also suggests potential role of other variables that affect LDHA expression possibly including cancer subtypes. Thus, we have also conducted an exemplified subtype analyses for medulloblastoma. Medulloblastoma was selected as a model for this analysis since specific cell-signaling aberrations provide distinct outcomes as well as cellular activities in 4 subtypes, including metabolism (Juraschka \& Taylor, 2019; Panosyan, Lin, Koster, \& Lasky, 2017). For example, the subtype with activation of WNT pathway is linked with better response to current treatments, and this effect presumably triumphs hypermetabolic aspects caused by an increased LDHA. Meanwhile, in the other 3 types, where response to current treatments generally is not as effective - LDHA expression shows possible inverse association with survival (Figure 5).

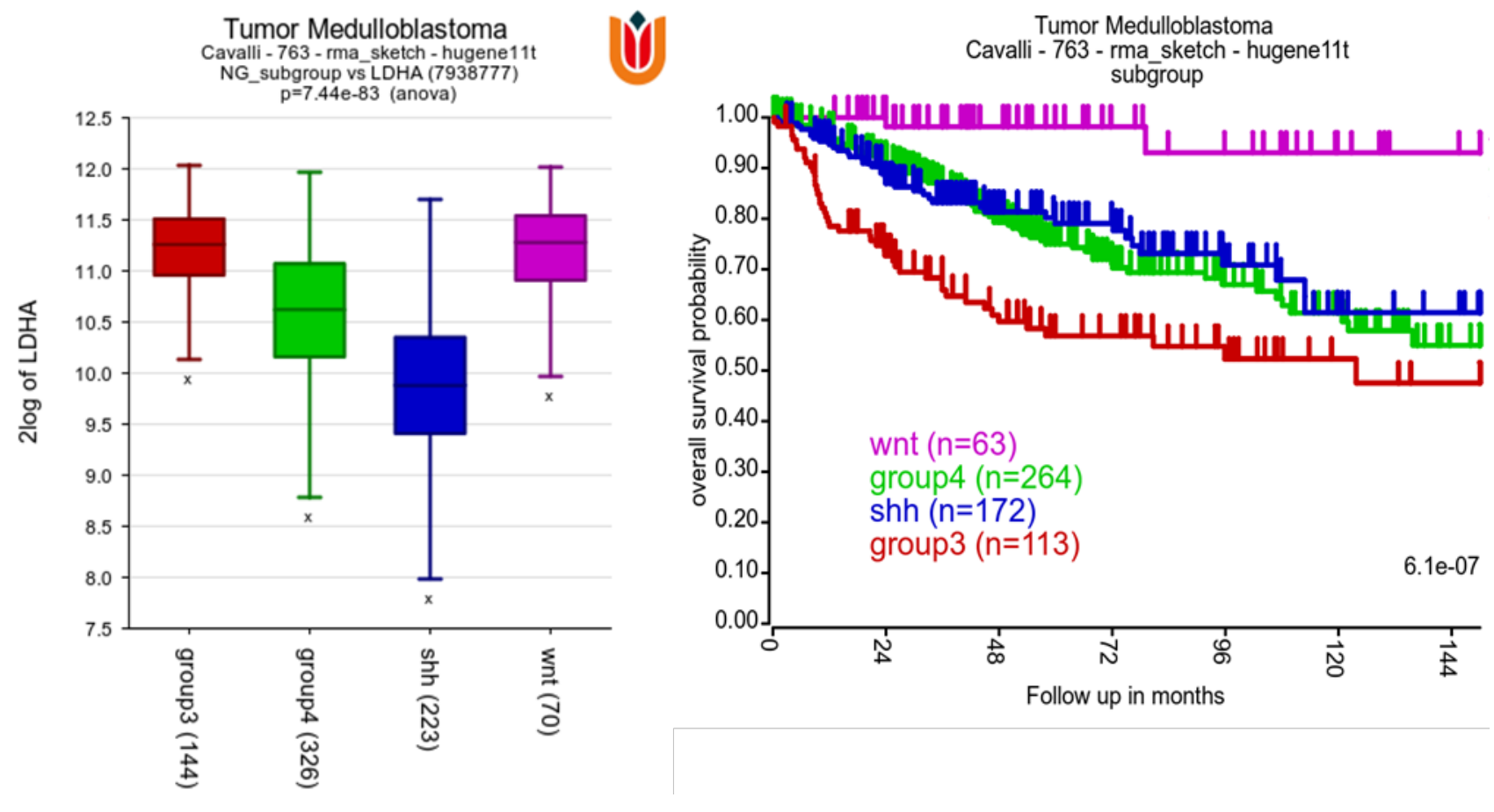

Figure 5. In 3 out of 4 medulloblastoma subtypes LDHA expression correlates with survival (http://r2.amc.nl). 
The largest medulloblastoma dataset in R2 was used to conduct the analyses of 4 molecular subtypes. All four subtypes were analyzed for LDHA expression (bar graphed panel on the left) and Kaplan-Meier analysis by categorical track (plots shown on the right side of the figure). With the exception of WNT subgroup that has very good prognosis, all the other 3 subtypes have poorer prognoses that match with relative expression of LDHA. Theoretical explanation is that specific signaling aberrations in WNT pathway are linked with better response to current treatments, and this effect triumphs presumed hypermetabolic state caused by increased LDHA. Whereas LDHA expression shows inverse association with survival in the other 3 types where response to current treatment generally is not as effective. This allows hypothesizing that an anti-metabolic approach targeting LHDA may have variable effects based on cancer-subtypes.

\section{Discussion cont.}

Although higher LDHA expression is observed in colon cancer and B-cell lymphomas, similar to WNT medulloblastomas it was associated with better clinical outcome, respectively in R2 datasets Xiao-420 and Guinney-3232. Interestingly, PET imaging is being increasingly used to adjust lymphoma treatments for improving clinical outcomes (ElGalaly et al., 2018). In fact, not all of the other cancers and lymphoma subtypes are "PET-avid or PET-positive" (ElGalaly et al., 2018), also indirectly supporting that Warburg effect may not be uniformly up-regulated in all cancers.

Nevertheless, larger groups of cancers showed significant overall association between the survival and expression of few key metabolic enzymes as shown in Figure 4 and Table 1. Limitations of our study included predominant use of a single molecule (LDHA) as an indirect mean of clinical evaluation of Warburg effect. To increase comprehensiveness of this study, last part of our work included 3 other key enzymes helping to demonstrate the oncometabolic heterogeneity of cancers.

Lastly, we propose that metabolic derangements can serve to diversify anti-metabolic strategies for developing more targeted therapies against different types of cancers. As a potential model of specific ant-metabolic therapeutics development we suggest consideration of the "antibody-drug conjugate" concept ("Antibody-drug Conjugate, a Potential Trojan Horse to Fight Cancer https://www.genscript.com/antibody-news/antibody-drug-conjugate-apotential-trojan-horse-to-fight-cancer.html," May 18, 2021). As shown in Figure 6 available LDHA inhibitor(s) (Feng et al., 2018) may be linked to monoclonal antibodies designed for selective binding to certain types of cancers. Hypothetically, selective targeting of the Warburg effect with LDHA inhibition inside the cancer cells may produce an effective anti-cancer effect. Normal tissues in this case should be relatively spared of LDHA inhibition, which can hopefully allow minimization of side effects. Further pre/clinical research would be crucial to address these theories. 


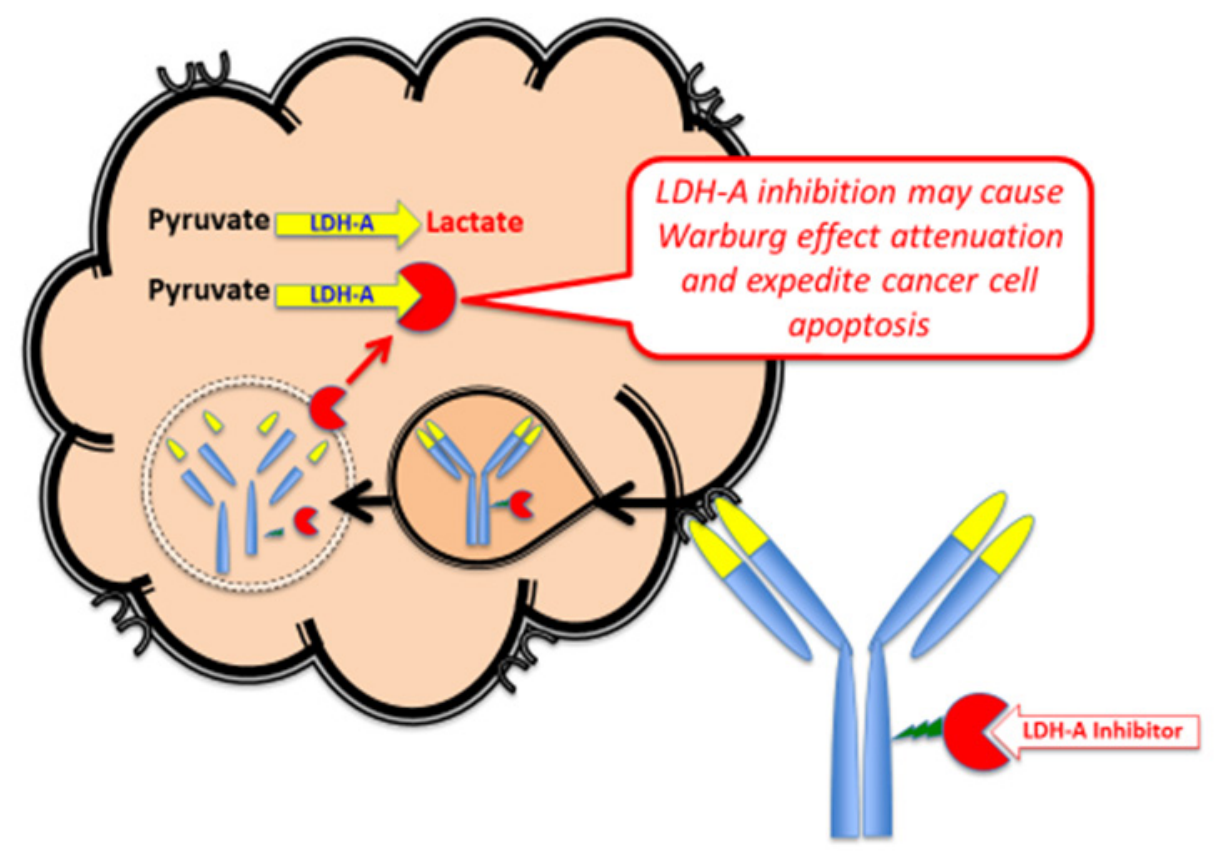

Figure 6. Proposed concept for additive antimetabolic cancer therapeutics with an antibody (Ab)-LDHA inhibitor conjugate (not to scale).

Cancer cell apoptosis maybe more effective with targeted delivery of a LDHA inhibitor (in red) linked with monoclonal $\mathrm{Ab}$ against a specific cancer. Light chain of the immunoglobulin (in yellow) is designed to have a high affinity for the molecules of an antigen, which predominantly or exclusively is expressed on the cancer cells. LDHAinhibitor linked with this $\mathrm{Ab}$ gets internalized as an endosome and the complex gets cleaved intracellularly. Molecules of the detached LDHA inhibitor then provide an enzymatic shutdown with blockage of lactate formation. Resulting attenuation of the Warburg effect may then expedite the cancer cell apoptosis, especially if coupled with other therapies. Similar concept can be applied for the other key enzymes of oncometabolism.

\section{Conclusion}

Warburg effect may not be universally dominant for all types of cancers, but most cancers have high LDHA expression and/or associated poor survival - confirming overall central role of this important metabolic derangement in cancers. Heterogeneity of metabolic alterations can serve to diversify anti-metabolic strategies for developing more targeted therapies against cancer types.

\section{Limitations}


Limitations of our study included predominant use of a single molecule (LDHA) as an indirect mean of clinical evaluation of Warburg effect. To increase comprehensiveness of this study, last part of our work included 3 other key enzymes helping to demonstrate the oncometabolic heterogeneity of cancers.

\section{Acknowledgments}

The results shown here are in whole or part based upon data generated by the R: Genomics Analysis and Visualization Platform (http://r2.amc.nl), and Human Protein Atlas available from http://www.proteinatlas.org (Atlas).

\section{References}

1. (http://r2.amc.nl), R. G. A. a. V. P. R2: Genomics Analysis and Visualization Platform (http://r2.amc.nl), (https://hgserver1.amc.nl/cgi-bin/r2/main.cgi), R2: Kaplan Meier Scanner (amc.nl) (https://hgserver1.amc.nl/cgibin/r2/main.cgi?option=kaplan_main).

2. Akella, N. M., Ciraku, L., \& Reginato, M. J. (2019). Fueling the fire: emerging role of the hexosamine biosynthetic pathway in cancer. BMC biology, 17(1), 52-52. doi: 10.1186/s12915-019-0671-3

3. Ananieva, E. A., \& Wilkinson, A. C. (2018). Branched-chain amino acid metabolism in cancer. Current opinion in clinical nutrition and metabolic care, 21(1), 64-70. doi: 10.1097/MCO.0000000000000430

4. Antibody-drug Conjugate, a Potential Trojan Horse to Fight Cancer https://www.genscript.com/antibodynews/antibody-drug-conjugate-a-potential-trojan-horse-to-fight-cancer.html. (May 18, 2021).

5. Atlas, T. H. P. The Human Protein Atlas (https://www.proteinatlas.org/humanproteome/pathology), (https://www.proteinatlas.org/ENSG00000134333-LDHA/pathology).

6. Avramis, V. I., \& Panosyan, E. H. (2005). Pharmacokinetic/Pharmacodynamic Relationships of Asparaginase Formulations: The Past, the Present and Recommendations for the Future. Clinical Pharmacokinetics, 44(4), 367-393.

7. Broome, J. D. (1963). Evidence that the L-asparaginase of guinea pig serum is responsible for its antilymphoma effects. I. Properties of the L-asparaginase of guinea pig serum in relation to those of the antilymphoma substance. The Journal of experimental medicine, 118(1), 99-120. doi: 10.1084/jem.118.1.99

8. El-Galaly, T. C., Villa, D., Gormsen, L. C., Baech, J., Lo, A., \& Cheah, C. Y. (2018). FDG-PET/CT in the management of lymphomas: current status and future directions. J Intern Med, 284(4), 358-376. doi:

10.1111/joim.12813

9. Ertel, I. J., Nesbit, M. E., Hammond, D., Weiner, J., \& Sather, H. (1979). Effective Dose of 1-Asparaginase for Induction of Remission in Previously Treated Children with Acute Lymphocytic Leukemia: A Report from Childrens Cancer Study Group. Cancer Research, 39(10), 3893-3896.

10. Feng, Y., Xiong, Y., Qiao, T., Li, X., Jia, L., \& Han, Y. (2018). Lactate dehydrogenase A: A key player in carcinogenesis and potential target in cancer therapy. Cancer medicine, 7(12), 6124-6136. doi: 10.1002/cam4.1820 
11. Hasawi, N. A., Alkandari, M. F., \& Luqmani, Y. A. (2014). Phosphofructokinase: A mediator of glycolytic flux in cancer progression. Critical Reviews in Oncology/Hematology, 92(3), 312-321. doi: https://doi.org/10.1016/j.critrevonc.2014.05.007

12. Juraschka, K., \& Taylor, M. D. (2019). Medulloblastoma in the age of molecular subgroups: a review. J Neurosurg Pediatr, 24(4), 353-363. doi: 10.3171/2019.5.peds18381

13. Koukourakis, M. I., \& Giatromanolaki, A. (2019). Warburg effect, lactate dehydrogenase, and radio/chemo-therapy efficacy. International Journal of Radiation Biology, 95(4), 408-426. doi: 10.1080/09553002.2018.1490041

14. Mukherjee, S. (16 November 2010). Emperor of All Maladies: A Biography of Cancer.

15. Panosyan, E. H., Lin, H. J., Koster, J., \& Lasky, J. L. (2017). In search of druggable targets for GBM amino acid metabolism. BMC Cancer, 17(1), 162. doi: 10.1186/s12885-017-3148-1

16. Potter, M., Newport, E., \& Morten, K. J. (2016). The Warburg effect: 80 years on. Biochemical Society transactions, 44(5), 1499-1505. doi: 10.1042/BST20160094

17. Valvona, C. J., Fillmore, H. L., Nunn, P. B., \& Pilkington, G. J. (2016). The Regulation and Function of Lactate Dehydrogenase A: Therapeutic Potential in Brain Tumor. Brain pathology (Zurich, Switzerland), 26(1), 317. doi: 10.1111/bpa.12299

18. Warburg, O., Wind, F., \& Negelein, E. (1927). THE METABOLISM OF TUMORS IN THE BODY. The Journal of general physiology, 8(6), 519-530. doi: 10.1085/jgp.8.6.519

19. Wilson, M., Cummins, C. L., MacPherson, L., Sun, Y., Natarajan, K., Grundy, R. G., . . Peet, A. C. (2013). Magnetic resonance spectroscopy metabolite profiles predict survival in paediatric brain tumours. European journal of cancer (Oxford, England : 1990), 49(2), 457-464. 